\title{
A CRITICAL EVALUATION OF THE CASE FOR COMPETITIVE SELECTION IN CEPAEA
}

\author{
WALLACE ARTHUR \\ Department of Biology, Sunderland Polytechnic, Sunderland SR1 3SD, U.K. \\ Received 18.xii.81
}

\section{SUMMARY}

\begin{abstract}
One of several populations showing an association between a low frequency of unbanded Cepaea nemoralis and the distribution of the congeneric species $C$. hortensis was surveyed in detail in an attempt to determine the selective agent causing the association. The sympatric area was characterized by greater vegetational cover and higher plant species diversity than the area occupied only by $C$. nemoralis, suggesting that snails in the latter area receive greater insolation. It is thus possible that a microclimatic variable gives rise, independently, to the restricted distribution of $C$. hortensis and the coincident area of predominantly banded $C$. nemoralis, rather than the first of these two effects causing the second. The possible extension of this conclusion to other populations is considered and it is seen that climatic selection could produce all the observed associations involving the banding morph-frequency in $C$. nemoralis. Although associations between banding morph-frequency in $C$. hortensis and the distribution of $C$. nemoralis are not readily explicable in terms of climatic selection, the case for a general association of this kind is weak. It is concluded that it is unnecessary to invoke competitive selection to explain the observed patterns of variation in morph-frequency, but that its existence cannot be ruled out. A suggestion is made regarding the choice of model system for future studies of a similar kind.
\end{abstract}

\section{INTRODUCTION}

THE evolutionary importance of interspecific competition, particularly its role, if any, as a source of selective pressure on individual polymorphisms, is much less certain than, for example, the importance of predators and climate. Predators have been implicated in altering gene-frequencies of the well known visual polymorphisms of Cepaea (Cain and Sheppard, 1950, 1954) and Biston (Kettlewell, 1956), and climate is known to be effective in altering the frequencies of Cepaea visual morphs (Jones et al., 1977) and of inversion karyotypes in Drosophila (Dobzhansky, 1943). It may also be important in determining the frequencies of some allozymic variants in a number of species (Koehn, 1969; Pipkin et al., 1973; Place and Powers, 1979). In contrast, there is not a single case in which interspecific competition can be clearly seen to cause spatial or temporal variation in the gene-frequency of a polymorphism in a natural population.

The visual polymorphisms of Cepaea provide a useful system upon which to base a search for "competitive selection"-a phrase which will be used here to denote selection arising from interspecific competition. The two British species of Cepaea, C. nemoralis and $C$. hortensis, have a similar range of phenotypes with similar genetic bases (see review by Murray, 1975). There are several lines of evidence for competition between these two species (Cameron, 1970b; Arthur, 1977) and, due to the small size and low mobility of Cepaea, a number of independent allopatric/sympatric comparisons of morph-frequency may be made within a reasonably small geographical area. 
In two previous papers (Arthur, 1978, 1980) I have shown that when comparisons of banding morph-frequency are made between neighbouring allopatric and sympatric areas, it is often found that the latter are characterized by a significant reduction in the frequency of unbanded shells. This is true of both species, though the evidence is stronger in the case of $C$. nemoralis. The reverse trend, that is, a significant increase in unbandeds in sympatry, was not found to occur in either of the species in any of the areas studied. One possible explanation for the pattern of spatial variation observed is that unbanded snails, of both species, are weaker interspecific competitors than bandeds (Arthur, 1978). The major weakness of this hypothesis, as has already been pointed out (Arthur 1978, 1982) is that there is no obvious mechanism whereby unbanded snails would be expected to be less able competitors. The need for an understanding of the mechanism of selection has been stressed by Clarke (1975) in the context of distinguishing which of a group of linked loci (or loci in linkage disequilibrium due to epistasis) is the subject of selection. This need is equally applicable when distinguishing which of a group of co-varying ecological factors constitutes a selective agent.

Because of the lack of an apparent mechanism for competitive selection, it was decided to re-survey one of the allopatry/sympatry transects in detail, examining a number of ecological variables, to determine whether an interpretation in terms of climatic selection, for which there is a known mechanism (Heath, 1975; Richardson, 1974), might be possible. The area chosen for intensive re-survey was the one that showed the strongest and most precise association between a low frequency of unbanded $C$. nemoralis and the distribution of $C$. hortensis. In the present paper the data resulting from this survey are analyzed and their general implications discussed.

\section{Methods AND AREA DESCRIPTION}

\section{(i) The survey area}

Snails were collected from an approximately linear series of 17 sampling sites on the dunes stretching between Seaton Sluice and Blyth, Northumberland. The locations of the samples are shown in fig. 1, the distance between samples 1 and 17 being around $1.8 \mathrm{~km}$. This is slightly greater than the distance between the extreme northern and southern sites in the previous survey (Arthur, 1978) as sampling was extended further northwards in the present study to include the entire dune system up to the point where it gives way to disturbed, then to lawned, areas on approaching Blyth.

\section{(ii) Sampling and scoring}

These were carried out as described in Arthur (1978), though samples were more widely spaced to reduce the problem of non-independence inherent in detailed surveys. The maximum sampling site diameter of $20 \mathrm{~m}$ remained unchanged.

\section{(iii) Precise locations of samples}

To facilitate any re-sampling of exactly the same sites in the future (an approach which has proved informative in other instances-Clarke and 


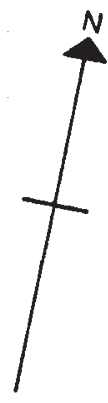

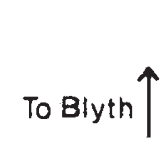

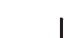

$\left|\begin{array}{l}\text { start of } \\ \text { lawned } \\ \text { area }\end{array}\right|$

17

$\stackrel{3}{\frac{9}{<}}$

Agricultural

land

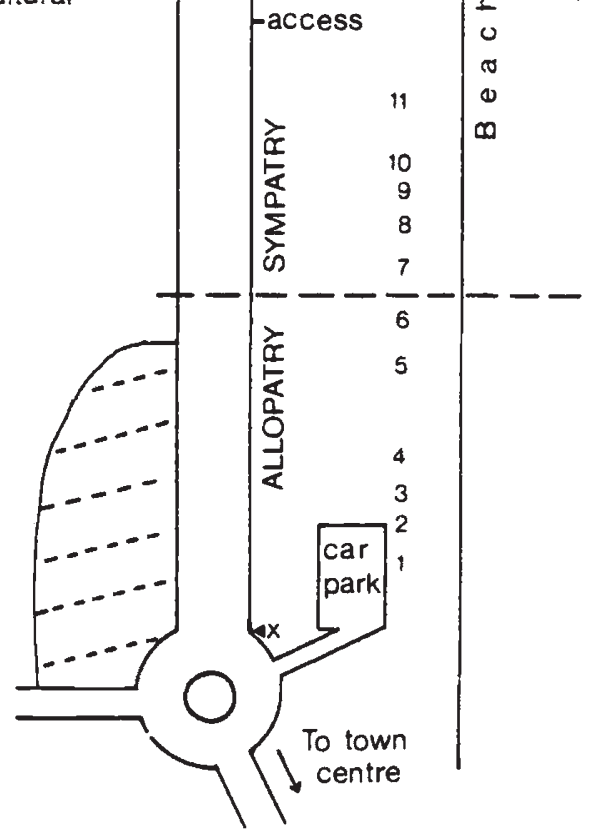

Fig. 1.-Diagram of area surveyed showing approximate locations of the 17 sampling sites. The significance of the point marked $X$ is explained in the Methods section. Shading indicates a built-up area.

Murray, 1962a, b; Wall et al., 1980) the sites have been located as accurately as possible in two ways. Firstly, their distance north of the large roundabout at the southern end of the dune system was determined by ascertaining which of a line of lamp-posts on the main road parallel to the 
dunes was opposite each sampling site. These values range from lamp-post "3.5" for sample 1 to lamp-post 64 for sample 17. The lamp-post at point " $X$ " on fig. 1 is numbered zero. This method of locating the samples in a North/South direction, which is accurate to about $\pm 10 \mathrm{~m}$, forms the $x$-axis of figs. 2, 4, 5 and 6. Secondly, the centres of all sampling sites have been pinpointed on a $3 \mathrm{ft}$ sq. aerial photograph (scale $1: 4000$ ) of the dune system. This photograph will be kept indefinitely at the Biology Department at Sunderland and made available to any future Cepaea workers interested in the Seaton Sluice population.

\section{(iv) Ecological variables}

The aerial photograph was also used to estimate the proportion of vegetational cover at each sampling area. This was estimated by measuring, to the nearest 5 per cent, the proportions of bare sand and vegetation (which are easily distinguished on the photograph as pale and dark patches with little intergradation) in an area equivalent to $20 \mathrm{~m} \times 20 \mathrm{~m}$ centred on the middle of the sampling site. Plant species diversity was estimated simply as the number of macroscopic plant species in each site, which was determined by searching each site with roughly equal intensity and identifying all species found. No attempt was made to assess relative abundance of the various plant species.

\section{Results}

(i) Persistence of the pattern of variation in $\mathrm{C}$. nemoralis

Before proceeding to examine other variables in an attempt to explain the variation in the frequency of unbanded $C$. nemoralis along the dunes, it was necessary to confirm that the pattern of variation revealed in the 1976 survey (Arthur, 1978) was not detectably modified in 1980, when the present study was conducted. This was particularly necessary since the 1976 samples were obtained in May immediately prior to the unusually hot, dry summer of 1976 which might have resulted in strong selection for paler shells such as unbandeds. The combined 1976 and 1980 data for $C$. nemoralis are shown in fig. 2 . It can be seen that there is close agreement in morph-frequency in adjacent samples from the two surveys. However, the small number of 1980 allopatric samples should also be noted: this point will be considered further in section (iv).

\section{(ii) Reliability of morph-frequency and species-frequency estimates}

It is impossible to subject the banding polymorphism in $C$. hortensis at Seaton Sluice to the same form of analysis as that used for C. nemoralis-i.e., comparison of allopatric and sympatric groups of samples-because there is no pure $C$. hortensis population on the dunes. However, in an earlier paper (Arthur, 1978) I showed that the frequency of unbanded $C$. hortensis declined, within the sympatric area, with increasing species-frequency of C. nemoralis. This latter form of analysis requires that the variable "speciesfrequency", that is, $N_{\text {nem }} /\left(N_{\text {nem }}+N_{\text {hort }}\right)$ can be satisfactorily measured. Repeat-sampling of a site in Derbyshire, described in the same paper, 


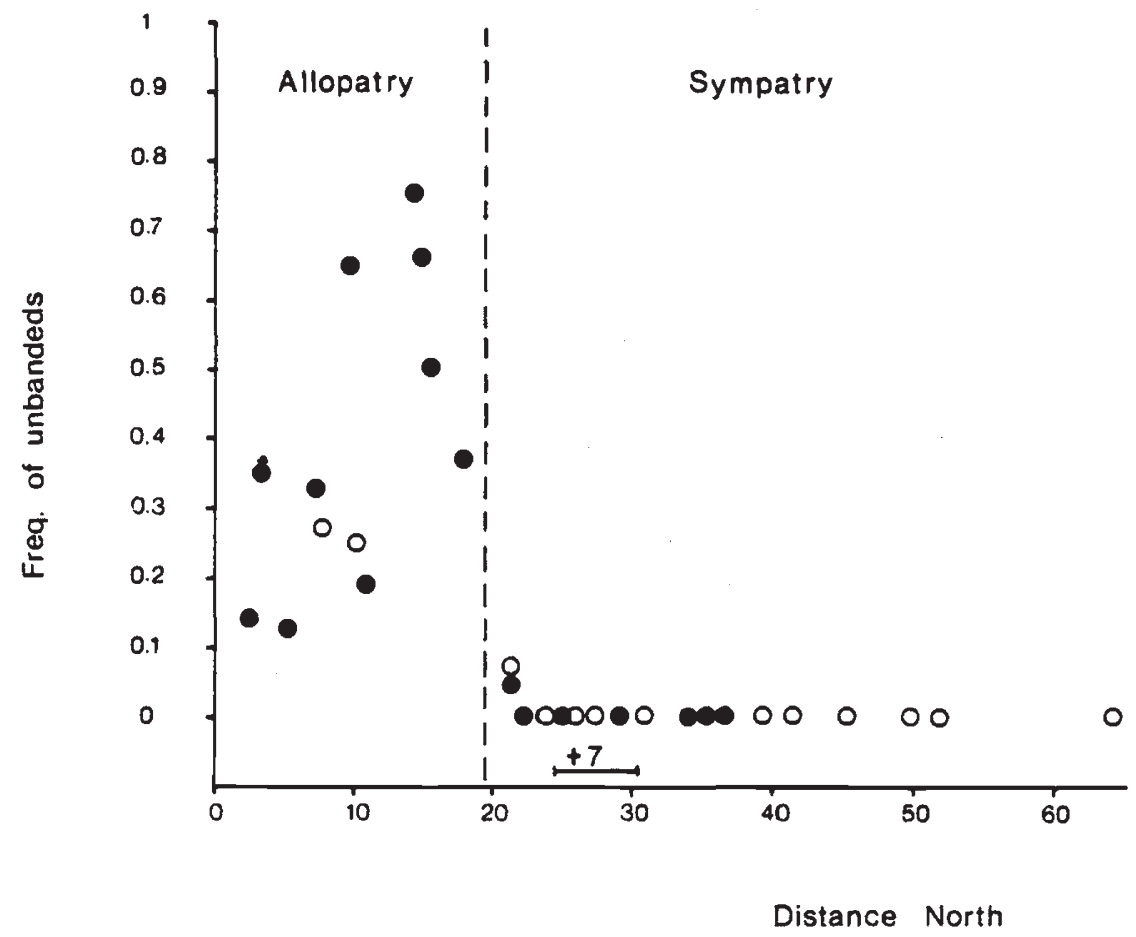

Fig. 2.-Variation in the frequency of unbanded $C$. nemoralis at Seaton Sluice. Open circles-1980 samples; closed circles-1976 samples. For explanation of distance units, see Methods section. The +7 in the figure indicates that there were 7 further 1976 samples in the area shown. All had a frequency of zero.

showed no significant difference between successive samples in speciesfrequency estimates. However, Clarke (1962) re-sampled a number of sites in the Oxford area and found highly significant differences in speciesfrequency at two of them. (Morph-frequency, on the other hand, was highly repeatable.) It should be noted, however, that both sites showing significant differences in species-frequency in Clarke's study were visited on two occasions approximately a year apart. This renders the significant result ambiguous, since the population dynamics of two separate species may be quite different, and thus it is not clear whether the different estimates of species-frequency reflect population changes or poor sampling reliability. Because of this problem, and the desirability of information on sampling reliability within the area involved in the present study, one of the mixedspecies sites at Seaton Sluice (site 8) was sampled on two occasions only three weeks apart. Significant differences in species-frequency estimates over a three-week period are almost certainly due to sampling error. The data on site 8 are shown in table 1, and it is clear that while morph-frequency is consistent, the species-frequency estimates differ very markedly. It is of interest to note that the difference in species-frequency is made up almost entirely of a difference in the numbers of $C$. hortensis on the two occasions, which depart very significantly from a 1:1 ratio, while for $C$. nemoralis the null hypothesis of equal abundance on the two occasions cannot be rejected. 
TABLE 1

Variation in morph-frequency and species-frequency estimates at site 8. B-number of bandeds; UB-number of unbandeds; N-total numbers

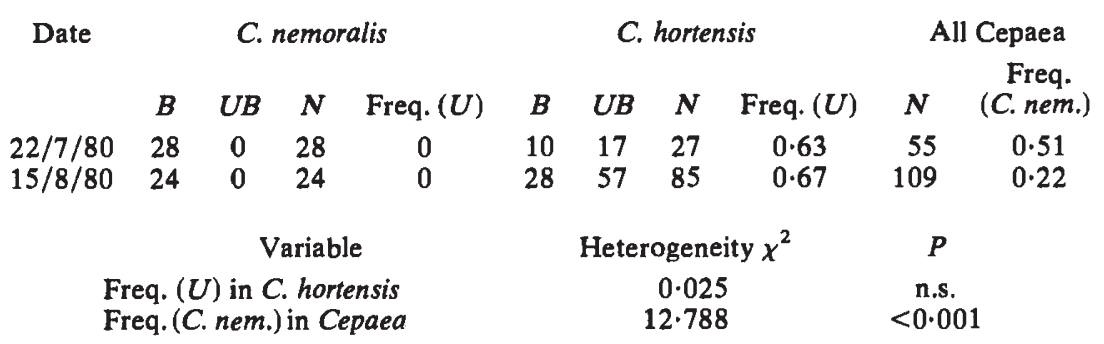

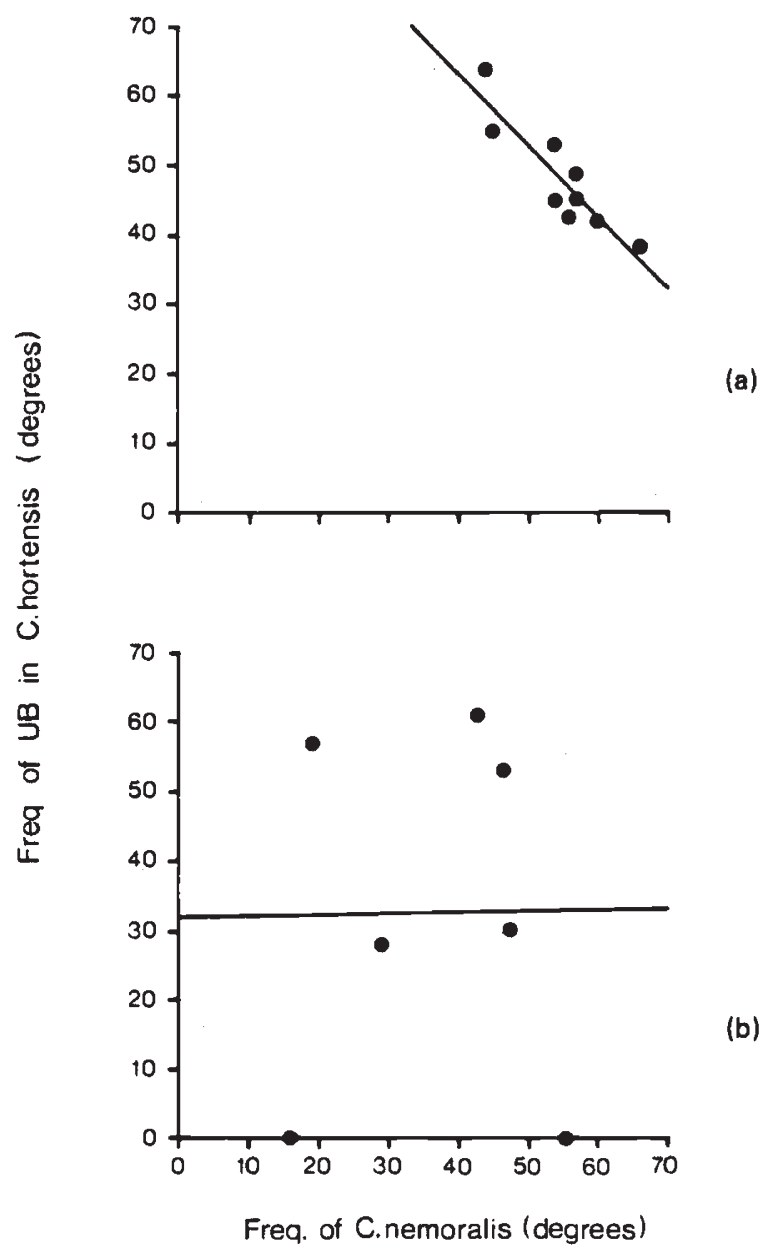

FIG. 3.-Relationship between the frequency of unbandeds in $C$. hortensis and the frequency of $C$. nemoralis in the sample in 1976 (a) and 1980 (b). Lines were obtained by linear regression; both variables are arcsin-transformed. Samples where $N<5$ for $C$. hortensis are omitted. $P$-values are for $t$-testing of calculated regression coefficients against a null hypothesis of $\beta=0.1976$ data: $p<0 \cdot 001,1980$ data: $p>0 \cdot 1$. 
Fig. 3 shows the relationship between the frequency of unbanded $C$. hortensis and the species-frequency of $C$. nemoralis in 1976 (fig. 3(a)) and 1980 (fig. 3(b)). Clearly, the observed relationships are discrepant. It is not clear whether the negative relationship shown in the 1976 data is an artifact produced by sampling errors or whether it exists and is obscured in 1980 by sampling errors; at any rate it seems unwise to accept the 1976 pattern as evidence for selection in $C$. hortensis, given that species-frequency cannot be reliably estimated. Consequently, the present paper is concerned largely with interpreting variation in $C$. nemoralis (but see also Discussion).

\section{(iii) Vegetational cover and plant species diversity}

C. nemoralis appears to be tolerant of a warmer micro- and macroclimate than $C$. hortensis. This is suggested by comparative behavioural (Cameron, 1970a), ecological (Cameron, 1970b) and geographical (Taylor, 1914) studies on the two species. It was thus suspected that, despite a superficial similarity of habitat in allopatric and sympatric sections of the dune system, the absence of $C$. hortensis in the former might be due to increased insolation at ground level. The most obvious way in which one sampling site would receive more insolation at ground level than another is if it consisted of a greater proportion of non-vegetated patches of bare sand. Figure 4 shows the variation in a cover index measuring the proportion of the site that is vegetated. (The data used in fig. 4, and in other figures, are given in table 2.) It can be seen from table 3 that the difference between allopatric and sympatric groups of samples is significant, allopatric samples

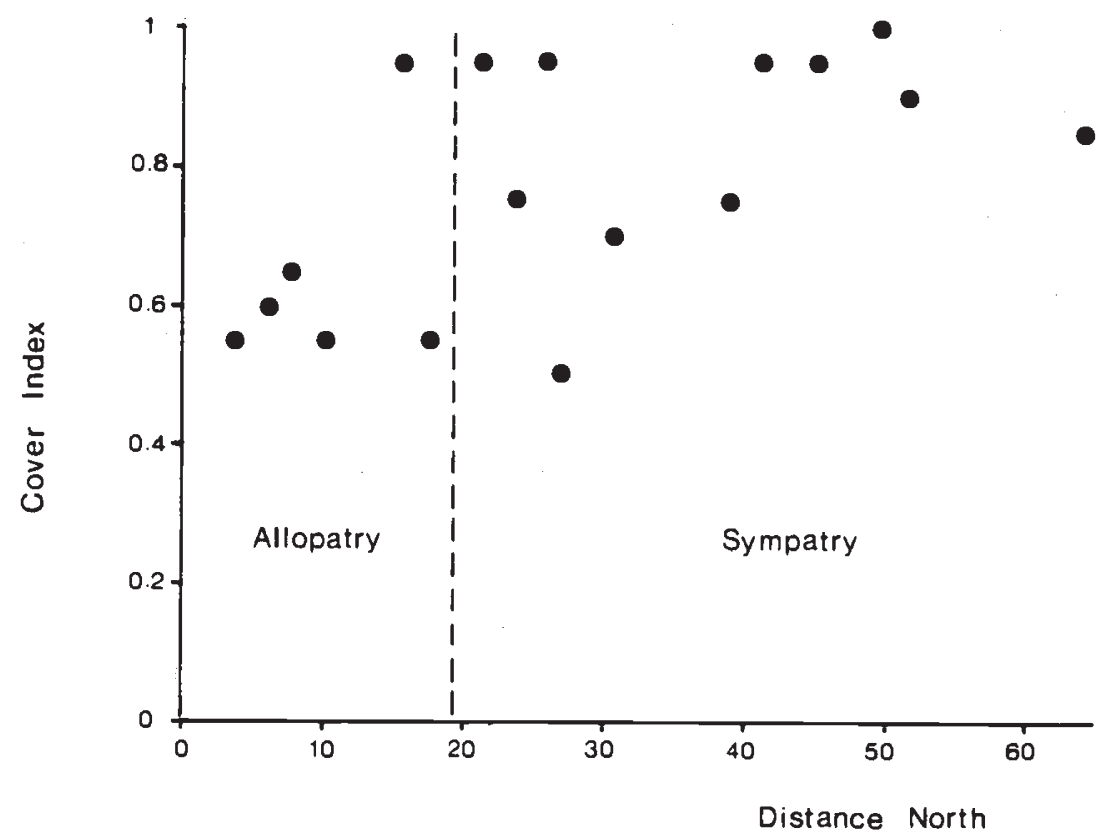

FIG. 4.-Variation in a vegetational cover index between sampling sites. See Methods for explanation of units along both axes. 


\section{TABLE 2}

Summary of data from 1980 survey at Seaton Sluice. For details of location and cover index units, see Methods section. Samples 1-6, allopatric area; samples 7-17, sympatric. Freq. (UB) figures are bracketed where $\mathrm{N}<10$ but are included to show that no unbandeds were found in the sympatric area

$\begin{array}{ccccccc}\begin{array}{c}\text { Sample } \\ \text { no. }\end{array} & \text { Location } & \begin{array}{c}\text { Cover } \\ \text { index }\end{array} & \begin{array}{c}\text { Plant } \\ \text { species }\end{array} & N \text { (live) } & \begin{array}{c}C \text {. nemoralis } \\ N \text { (total) }\end{array} & \text { Freq. (UB) } \\ 1 & 3.5 & 0.55 & 10 & 0 & 0 & - \\ 2 & 6.0 & 0.60 & 11 & 0 & 0 & - \\ 3 & 7.5 & 0.65 & 7 & 0 & 15 & 0.27 \\ 4 & 10.0 & 0.55 & 18 & 0 & 12 & 0.25 \\ 5 & 15.5 & 0.95 & 22 & 0 & 0 & - \\ 6 & 17.5 & 0.55 & 22 & 0 & 0 & - \\ 7 & 21.0 & 0.95 & 24 & 13 & 14 & 0.07 \\ 8 & 23.5 & 0.75 & 23 & 28 & 28 & 0 \\ 9 & 25.5 & 0.95 & 27 & 13 & 15 & 0 \\ 10 & 27.0 & 0.50 & 20 & 4 & 4 & (0) \\ 11 & 30.5 & 0.70 & 25 & 10 & 11 & 0 \\ 12 & 39.0 & 0.75 & 36 & 8 & 8 & (0) \\ 13 & 41.0 & 0.95 & 30 & 0 & 1 & (0) \\ 14 & 45.0 & 0.95 & 21 & 10 & 10 & 0 \\ 15 & 49.5 & 1.00 & 33 & 20 & 21 & 0 \\ 16 & 51.5 & 0.90 & 23 & 14 & 14 & 0 \\ 17 & 64.0 & 0.85 & 16 & 10 & 12 & 0\end{array}$

having less vegetational cover and thus, presumably, greater insolation at ground level.

Although live, active snails were observed on bare sand, up to $3 \mathrm{~m}$ from the nearest vegetation, the vast majority of snails can be found, at any one time, within clumps of vegetation. Thus variation between sampling sites in insolation within their vegetated component is likely to be of greater importance than variation in insolation between the sites at a more macroscopic level. This is unfortunately rather difficult to measure; however, it is likely to be related to plant species diversity for the following reason. All samples contained a large proportion of marram grass, Ammophila arenaria, which, because of its approximately vertical growth-form allows

TABLE 3

Comparison of allopatric and sympatric areas for a number of variables. $\mathrm{n}_{1}$-number of allopatric samples; $\mathrm{n}_{2}$-number of sympatric samples. Usmaller version of the Mann-Whitney U statistic. The larger version, which is sometimes tabulated instead, can be obtained from the formula $\mathbf{U}^{\prime}=$ $\left(\mathrm{n}_{1} \mathrm{n}_{2}-\mathrm{U}\right)$. Note that 0.002 is the lowest two-tailed probability level given in widely-available tabulations of $\mathrm{U}$

Variable

$\begin{array}{lrrrc} & n_{1} & n_{2} & U & P \\ 6 & 11 & 13 & =0.05 \\ & 6 & 11 & 7 & =0.01 \\ & 6 & 11 & 3 & <0.002 \\ & 10 & 14 & 0 & <0.002 \\ \text { d } & 2 & 11 & 0 & =0.05\end{array}$

Cover index

Plant species

No. of live

C. nemoralis

Freq. of unbanded

C. nemoralis (1976)

Freq. of unbanded

C. nemoralis (1980) 
considerable penetration of insolation. Additional species were mostly broad-leafed angiosperms, some of the commonest being dandelions, Taraxacum officinale agg. and the umbellifer Heracleum sphondylium. These provided shaded microhabitats under their leaves where in fact most Cepaea were found. Thus the effective insolation at a sampling site is likely to depend both on the number of non-marram species and on their abundances. Although it appeared from casual observation that these two variables were correlated-i.e., the more non-marram species, the greater their individual abundances-only the former was measured due to the large errors involved in estimating the abundances of many plant species in heterogeneous areas of up to $400 \mathrm{~m}^{2}$. The variation in plant species diversity is shown in fig. 5; as can be seen, samples from the allopatric area tend to have a lower number of plant species than those from the area of sympatry, and this difference is significant (table 3 ).

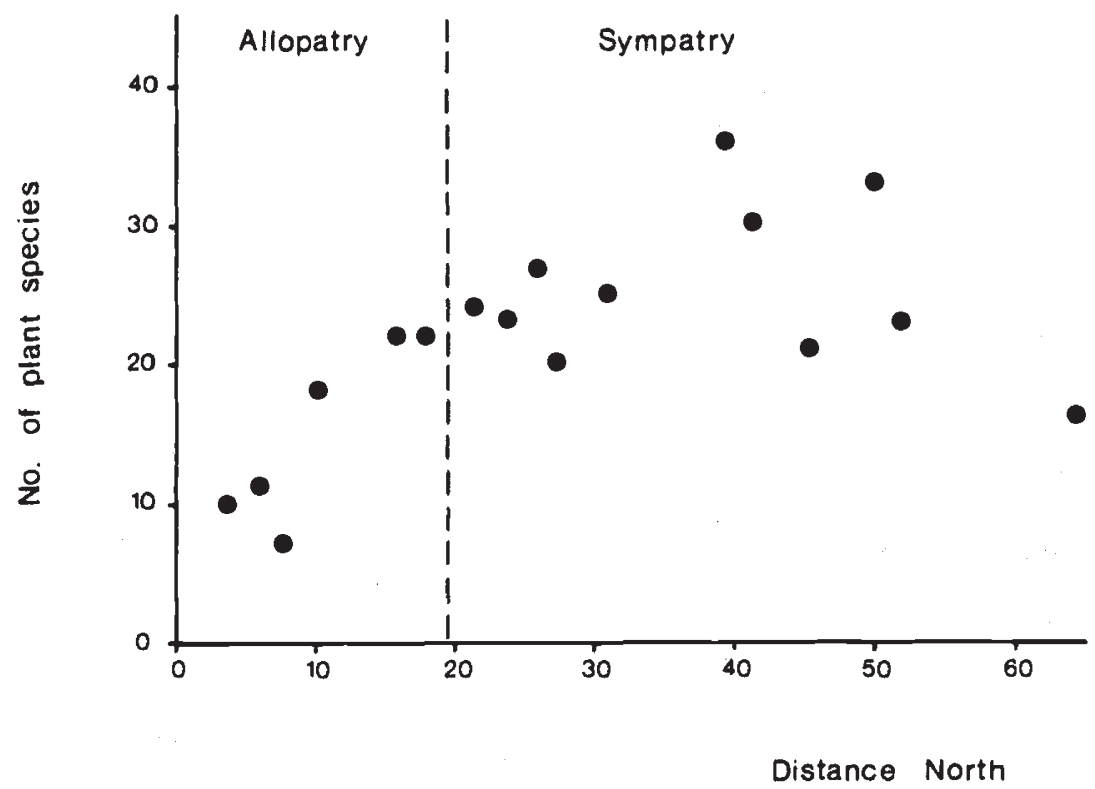

FIG. 5.-Variation in plant species diversity between sampling sites.

(iv) Relative abundance of C. nemoralis in 1980

Although the allopatric area has a significantly lower amount of vegetational cover and significantly fewer plant species than the sympatric area, there is no abrupt change in either variable at the allopatry/sympatry border, as occurs with the frequency of unbanded $C$. nemoralis. However, these vegetational measures are crude estimators of the amount of insolation received by the Cepaea population of a site. A much better estimate would be provided by the use of light-sensitive paint which can be applied to the shells (J. S. Jones, personal communication) but this method is precluded at present due to a lack of snails in the allopatric area. However, this lack in itself may be informative in relation to insolation. Since the 
generation time of Cepaea is about 3-4 years, the live snails found in 1980 represent the survivors of the 1976 heat wave plus their first-generation progeny. The variation in the number of live adult $C$. nemoralis obtained from similar sampling intensity at approximately equal-sized sites is shown in fig. 6. (Note that the two allopatric morph-frequency estimates shown

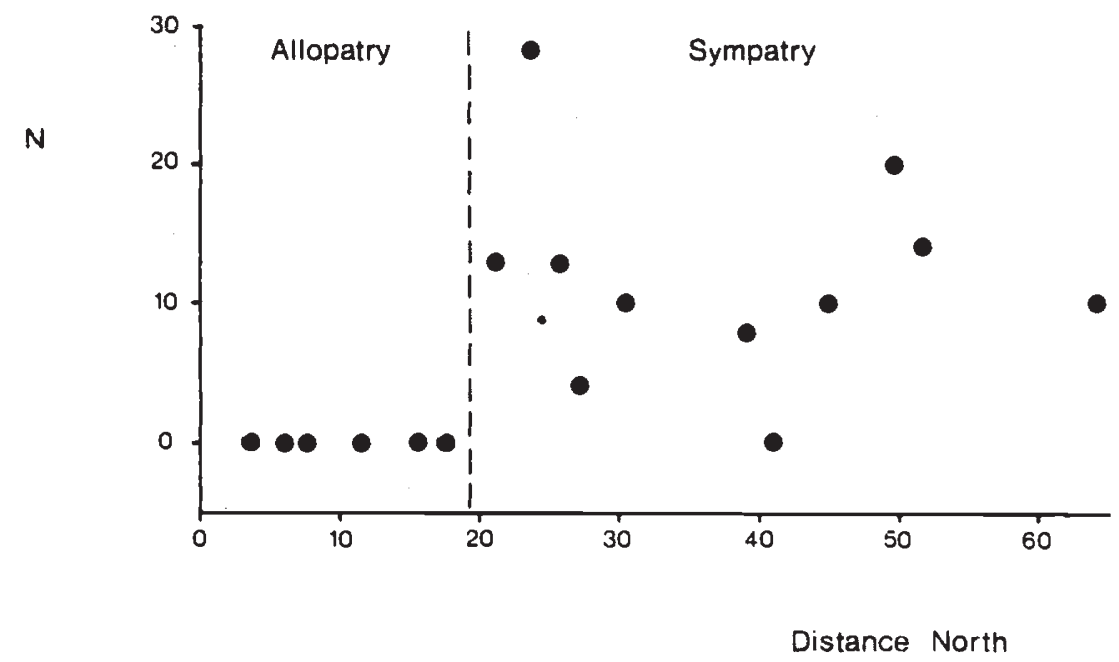

FIG. 6.-Variation in the number of live $C$. nemoralis found at different sampling sites in the 1980 survey.

in fig. 2 are based entirely on empty shells found at those sites-see table 2.) Further searches for live Cepaea in the allopatric area both within and outside of the specified sampling sites did not produce a single live snail, adult or juvenile. In contrast, live snails of both species were observed, in the sympatric area, both on subsequent visits to the specified sampling sites and at many other localities between the sites. The difference in relative abundance of $C$. nemoralis between the allopatric and sympatric areas is abrupt (fig. 6) and highly significant (table 3). This contrasts sharply with the situation in 1976 (see Arthur, 1978) when the mean sample sizes of C. nemoralis were 25.4 and 22.4 for the allopatric and sympatric areas respectively, the vast majority of snails in the 1976 survey being live adults.

\section{Discussion}

(i) The association between morph-frequency in $\mathrm{C}$. nemoralis and the distribution of C. hortensis at Seaton Sluice

The data presented in the previous section show that an explanation of the pattern of variation in $C$. nemoralis in terms of climatic selection is not unreasonable. The allopatric area, by comparison with the sympatric, is less densely vegetated and contains fewer plant species. This almost certainly means that snails receive greater insolation in the allopatric area. That this will produce selection in favour of unbanded shells is strongly suggested by the work of Heath (1975), who showed that banded and 
unbanded shells differed significantly in the equilibrium temperature they attained during a period of direct insolation. This differential heat-absorption effect is likely to be maximized in the populations of $C$. nemoralis at Seaton Sluice because all unbanded shells, whether yellow, pink or brown, were very pale in colouration, while the majority of bandeds were heavily fused, with many shells being almost completely black. The existence of a differential in heat-absorption does not necessarily imply a differential in survival, i.e., selection. However, Richardson (1974) presented evidence, from a natural population of $C$. nemoralis, that under strong insolation darker shells suffered a heavier mortality than paler ones, although it should be pointed out that this study involved the colour, rather than the banding locus.

An objection which might be raised against an explanation of the data in terms of climatic selection is the contrast between the gradual changes in vegetation along the dunes and the abrupt alteration in the banding polymorphism, from an area characterized by mid-range morph-frequencies to an entirely monomorphic banded area. However, there is no shortage of explanations for a slight and/or gradual environmental change leading to a large, stepwise change in gene-frequency and phenotype-frequency. Firstly, there is the possibility of interactions between loci resulting in the formation of steps in an originally gradual cline (Clarke, 1966). Secondly, when indirect environmental measures are employed, such as the use of vegetational indices to infer microclimatic changes, gradual alteration in the observed variable may mask stepwise changes in the associated variable that is of interest. Thirdly, a slight environmental change can cause a polymorphism to give way to monomorphism if it is initially balanced (at any mid-range frequency) by very weak heterotic or frequency-dependent forces; i.e., large changes in gene-frequency from some finite value to zero do not necessarily imply strong selection.

Since the pattern of variation in the frequency of unbanded $C$. nemoralis at Seaton Sluice is potentially explicable in terms of climatic selection, for which there is a known mechanism, it is unnecessary to invoke an additional selective process of unknown mechanism, e.g., some form of competitive selection, though the existence of unknown selective mechanisms cannot, of course, be ruled out.

\section{(ii) The general association between morph-frequency and coexistence in Cepaea}

It was mentioned in a previous paper (Arthur, 1980) that the parallel changes in morph-frequency at allopatry/sympatry borders in several geographical areas were most parsimoniously explained by a competitive selection hypothesis. However, the most parsimonious hypothesis is not always the correct one, and indeed Jones et al. (1977), in reviewing the Cepaea visual polymorphisms, have gone so far as to suggest that each separate population may require a unique explanation. Also, the difference in the explanatory power of climatic and competitive selection hypotheses, in relation to morph-frequency/coexistence associations, is not as great as first appears. If the interspecific difference in temperature tolerance mentioned above is a general phenomenon, then it might be expected that pure C. nemoralis populations will often occupy slightly warmer localities than 
neighbouring mixed-species colonies. Thus a general tendency for a low frequency of unbanded $C$. nemoralis to be associated with areas of coexistence, and a higher frequency to occur in allopatric populations, would be expected on the basis of climatic selection.

The main difference, then, between climatic and competitive selection hypotheses is that the latter potentially explains the morphfrequency/coexistence associations in both species (and in the single case in the related species Arianta arbustorum: see Arthur, 1980) whereas only the alterations in morph-frequency in $C$. nemoralis are readily explicable in terms of climatic selection. It is thus pertinent to re-examine the evidence for a general association between morph-frequency and coexistence in $C$. hortensis. This has already been started in section 3(ii) where it was seen that the evidence from the populations at Seaton Sluice was unreliable. Overall, I reported four associations between morph-frequency and coexistence in $C$. hortensis (Arthur, 1980). Of the remaining threediscounting the unreliable Seaton Sluice data-one was slightly outside formal significance $(p=0.08)$ and so may not represent a real effect. The other two associations (in Somerset and Hiddensee populations) cannot easily be dismissed. In both, there is a highly significant alteration in morph-frequency on entering sympatry, with the changes in morphfrequency coinciding quite precisely with the boundary between allopatry and sympatry. It is possible that these require unique local explanations, especially in the case of the Hiddensee population, since that survey was based upon a different genetic variable than the others (an index of band fusions rather than the frequency of unbandeds). Certainly, it is difficult to see how these two associations could be explained in terms of climatic selection.

In summary, then, although the hypothesis that unbanded snails are weaker interspecific competitors than bandeds is potentially capable of explaining all the trends in morph-frequency observed, it is seriously weakened by the lack of any apparent mechanism. In contrast, there is an obvious mechanism by which climatic selection may operate on the banding polymorphism in Cepaea. Although climatic selection cannot provide an explanation for all the data, it may well explain all of the associations between morph-frequency and coexistence in $C$. nemoralis; and the case for a general morph-frequency/coexistence association in $C$. hortensis is weak.

\section{(iii) Choice of model systems for the investigation of competitive selection}

Whether the lack of clear evidence for competitive selection on polymorphic variation is due to a lack of widespread competition, to a lack of evolutionary effects of competition on this category of variation, or simply to an insufficient number of thorough studies in this area, remains obscure. The speed at which this problem is resolved will depend critically on the choice of model system-i.e., upon which species and which genetic variables future studies are based. Although Cepaea has a number of advantages in this respect, as outlined in the introduction, it also has a serious drawback: the generation time is too long to permit successful selection experiments except, perhaps, where selective coefficients are extremely high. Since the distributions of competitors will in most cases be strongly associated with 
other ecological factors, it is essential to be able to perform selection experiments in the laboratory where presence/absence of a competitor and other variables such as temperature can be varied one at a time. It is strongly recommended that this is borne in mind at the outset, that is, at the stage when a model system is being chosen, in any future studies of the influence of interspecific competition on polymorphic variation.

Acknowledgments. - I am grateful to Dr John Cooke for identifying the plant species and to Miss Susan Harrison for typing the manuscript.

\section{REFERENCES}

ARTHUR, w. 1977. Studies on the relationship between environmental heterogeneity, natural selection and interspecific competition. $\mathrm{Ph} . \mathrm{D}$. Thesis, University of Nottingham.

ARTHUR, W. 1978. Morph-frequency and coexistence in Cepaea. Heredity, 41, 335-346.

ARTHUR, W. 1980. Further associations between morph-frequency and coexistence in Cepaea. Heredity, 44, 417-421.

ARTHUR, W. 1982. The evolutionary consequences of interspecific competition. Adv. Ecol. Res., 12, 127-187.

CAIN, A. J., AND SHEPPARD. P. M. 1950. Selection in the polymorphic land snail Cepaea nemoralis. Heredity, 4, 275-294.

CAIN, A. J., AND SHEPPARD, P. M. 1954. Natural selection in Cepaea. Genetics, 39, 89-116.

CAMERON, R. A. D. 1970a. The effect of temperature on the activity of three species of helicid snail (Mollusca: Gastropoda). J. Zool. London, 162, 303-315.

CAMERON, R. A. D. $1970 \mathrm{~b}$. Differences in the distributions of three species of helicid snail in the limestone district of Derbyshire. Proc. Roy. Soc. B, 176, 131-159.

CLARKE, B. 1962. Natural selection in mixed populations of two polymorphic snails. Heredity, $17,319-345$.

CLARKE, B. 1966. The evolution of morph-ratio clines. Am. Nat., 100, 389-402.

CLARKE, B. 1975. The contribution of ecological genetics to evolutionary theory: detecting the direct effects of natural selection on particular polymorphic loci. Genetics 79 Suppl., 101-113.

CLARKE, B., AND MURRAY, J. 1962a. Changes of gene-frequency in Cepaea nemoralis (L.). Heredity, 17, 445-465.

CLARKE, B., AND MURRAY, J. 1962 b. Changes of gene-frequency in Cepaea nemoralis (L.); the estimation of selective values. Heredity, $17,467-476$.

DOBZHANSKY, T. 1943. Genetics of natural populations. IX. Temporal changes in the composition of populations of Drosophila pseudoobscura. Genetics, 28, 162-186.

HEATH, D. J. 1975. Colour, sunlight and internal temperatures in the land-snail Cepaea nemoralis (L.). Oecologia, 19, 29-38.

KETTLEWELL, H. B. D. 1956. Further experiments on industrial melanism in the Lepidoptera. Heredity, 10, 287-301.

KOEHN, R. K. 1969. Esterase heterogeneity: Dynamics of a polymorphism. Science, 163, 943-944.

JONES, J. S., LEITH, B. H., AND RAWLINGS, P. 1977. Polymorphism in Cepaea: A problem with too many solutions? Ann. Rev. Ecol. Syst., 8, 109-143.

MURRAY, J. 1975. The genetics of the mollusca. In King, R. C. (ed.) Handbook of Genetics, vol. 3, Plenum, New York.

PIPKIN, S. B., RHODES, C., AND WILLIAMS, N. 1973. Relation of temperature to the frequency of the Drosophila alcohol dehydrogenase allele $A d h^{\text {II }}$. Genetics, 74, s213.

PLACE, A. R., AND POWERS, D. A. 1979. Genetic variation and relative catalytic efficiencies: lactate dehydrogenase $B$ allozymes of Fundulus heteroclitus. Proc. Nat. Acad. Sci. U.S.A., 76, 2354-2358.

RICHARDSON, A. M. M. 1974. Differential climatic selection in natural populations of the land snail Cepaea nemoralis. Nature, 247, 572-573.

TAYLOR, J. W. 1914. Monograph of the Land and Freshwater Mollusca of the British Isles, vol. 3. Taylor Bros., Leeds.

WALL, S., CARTER, M. A., AND CLARKE, B. 1980. Temporal changes of gene frequencies in Cepaea hortensis. Biol. J. Linn. Soc., 14, 303-317. 